\title{
Physician treatment of metastatic triple-negative breast cancer in the immuno-oncology era: a discrete choice experiment
}

\author{
Bruce Feinberg*,1(i), Skyler Hime" Jeff Wojtynek², Igoni Dokubo², Ajeet Gajra1', Yolaine \\ Smith ${ }^{1}$ \& Jonathan Kish ${ }^{1}$ \\ ${ }^{1}$ Cardinal Health Specialty Solutions Dublin, OH 60173, USA \\ ${ }^{2}$ Athenex, Inc., Buffalo, NY 60173, USA \\ *Author for correspondence: bruce.feinberg@cardinalhealth.com
}

\begin{abstract}
Aim: Guidelines list atezolizumab with nab-paclitaxel (ANP) as the preferred first-line (1L) therapy for metastatic triple-negative breast cancer (mTNBC) with PD-L1 expression $\geq 1 \%$, but which clinical attributes impact ANP prescribing? Materials \& methods: Medical oncologists participated in a discrete choice experiment (DCE) with four hypothetical mTNBC clinical scenarios to assess influences of: PD-L1 expression, menopausal status, prior adjuvant therapy and bulky liver metastases. Results: A total of $47 \%$ chose ANP in $1 \mathrm{~L}$ irrespective of menopausal status, prior adjuvant therapy or tumor bulk. PD-L1 expression was the only attribute with a significant impact on ANP preference, with $69 \%$ choosing ANP for those with $\geq 1 \%$ expression versus only $26 \%$ for those with $<1 \%$ ( $p<0.00001)$. Conclusion: ANP choice for $1 \mathrm{~L}$ mTNBC deviated from guidelines.
\end{abstract}

Lay abstract: Aim: Doctors aspire to practice evidence-based medicine, but the complexities of modern healthcare in which conflicting evidence, patient preferences, payer benefit design, financial toxicity and social determinants of health often challenge that pursuit. This is particularly true in the treatment of metastatic breast cancer (mBC). Materials \& methods: To better understand the influence of treatment of triple-negative $\mathrm{mBC}$, we designed a discrete choice experiment to examine the influence of patient age, disease severity, prior treatment and biomarkers on the choice for the recently approved novel treatment, atezolizumab with nab-paclitaxel (ANP), on triple-negative mBC. Results: ANP choice was relatively high, but unlike the traditional choices of single agent or combination chemotherapy which were influenced by age, disease severity and prior treatment, ANP choice was only influenced by biomarkers. Conclusion: ANP appears to have been rapidly adopted and is the preferred treatment across varied disease presentations. Biomarker influence on its choice is noteworthy but inconsistent across our survey sample.

First draft submitted: 20 July 2020; Accepted for publication: 1 September 2020; Published online: 21 September 2020

Keywords: clinical attributes $\bullet$ discrete choice experiment $\bullet$ mTNBC

The practice of evidence-based medicine (EBM), particularly regarding cancer chemotherapy, is somewhat aspirational as clinical and nonclinical influences on physicians and patients impact treatment choice. Breast cancer, as an example, is a heterogeneous disease with an increasing number of therapeutic options. Selecting an initial systemic treatment for a metastatic breast cancer $(\mathrm{mBC})$ patient is a complex process in which myriad factors must be considered, including:

- Tumor-specific factors: HR and HER2 status, genomic profile and biomarker expression, for example, PD-L1, $B R C A$;

- Disease-specific factors: stage at presentation, sites of disease and volume of tumor;

Future 8 Medicine 
- Clinical factors: prior adjuvant therapy, extent of previous surgical resection and radiation, nature of disease progression and time to relapse;

- Patient characteristics: age, comorbidities, menopausal status, psychological and sociological factors, patient preferences;

- Nontumor, nonclinical and nonpatient factors: physician preferences based on training and/or experience, behavioral economic heuristics, for example, status quo, personal/practice financial implications of treatment selection, payer constraints, for example, pathways and prior authorizations, social determinants of health, for example, distance from clinic, licensed driver, lives alone, among others.

An understanding of all these variables as well as knowledge of, and experience with, different therapeutic options may be necessary to establish optimal treatment sequences and outcomes for $\mathrm{mBC}$ patients [1-5].

Given the above-noted complexities, professional societies have established clinical guidelines for breast cancer, including, but not limited to, National Comprehensive Cancer Network, European Society of Medical Oncology - Advanced Breast Cancer 2, Spanish Society of Medical Oncology, Spanish Breast Cancer Research Group and American Society of Clinical Oncology [1]. These guidelines distinguish breast cancer by molecular subtype that can be abbreviated to three clinically relevant cohorts: those that overexpress HER2 (HER2+), those that are not HER2+ but hormone sensitive (HR+/HER2-) and those that are neither HER2+ nor HR+/HER2- and deemed triple negative. While triple-negative breast cancer (TNBC) accounts for $10-20 \%$ of all breast cancer, it represents a greater proportion of $\mathrm{mBC}$ as it has a poorer prognosis. Treatment guidelines recently added immuneoncology (IO) drugs and now list atezolizumab with nab-paclitaxel (ANP) as a preferred first-line (1L) treatment for metastatic TNBC (mTNBC) with PD-L1 expression $\geq 1 \%$. Prescribing behavior for this novel therapy, the impact of common clinical presentations and its influence on physician preferences in subsequent lines of therapy (LOT), second line $(2 \mathrm{~L})$, third line $(3 \mathrm{~L})$ have not been studied.

A literature search did not uncover a compelling validated instrument to address physician choice in prescribing chemotherapy. Randomized trial determinants of efficacy and toxicity, guideline preference (e.g., National Comprehensive Cancer Network) and practice- or payer-mandated clinical pathway dominate the literature as explanations for prescribing choice. Our intent was to examine physician choice beyond these more conventional determinants. Originally applied in social and economic research, discrete choice experiments (DCEs) have recently been applied to medical issues to assess respondents' preferences for different healthcare interventions. Hypothetical scenarios are presented to respondents who are asked to choose between two or more options. Options are defined by specific attributes with the relative importance of the attributes determined and quantified [6]. Paradoxically, few published DCE studies have investigated physicians' preferences as most have been conducted from patients' perspectives [7-9]. We conducted a DCE in which common $\mathrm{mBC}$ patient clinical scenarios (CS) were presented as case studies to participating physicians who were queried regarding choice of ANP in $1 \mathrm{~L}$ and the impact of $1 \mathrm{~L}$ choice on $2 \mathrm{~L}$ and $3 \mathrm{~L}$ treatment sequence, as well as explanations for that sequencing choice. The objectives of this study were: to determine the influence of patient, clinical and tumor factors (menopause status, prior adjuvant therapy, disease burden and PD-L1 expression) on the preference for ANP in 1L TNBC; to analyze the influence of $1 \mathrm{~L}$ treatment choice on subsequent systemic therapy sequencing; and to explain the reasons behind said choices $[3,10]$.

\section{Materials \& methods}

\section{Participant selection \& study design}

Physicians from the Cardinal Health Oncology Provider Extended Network were recruited to participate in this experiment and complete a web-based survey. To be eligible to participate, the physician had to be an oncologist/hematologist actively practicing in a community-based setting and have clinical experience treating mTNBC patients.

We conducted a DCE in which common patient CS were presented as case studies. The CS comprised four hypothetical patients with $\mathrm{mTNBC}$ with varying clinical attributes, including menopause status, prior adjuvant therapy, bulky disease and PD-L1 expression (Table 1). Physicians' choice of $1 \mathrm{~L}$ treatment, ANP versus standard chemotherapy, either single agent (SA) or combination chemotherapy (CC) other than ANP, for each of the four CS presented was recorded. We then extended those CS with uniform features of disease progression to assess $2 \mathrm{~L}$ and $3 \mathrm{~L}$ treatment preference patterns as they related to $1 \mathrm{~L}$ treatment choice (Table 1). An explanation was requested to describe the rationale for each treatment preference, for example, guideline recommended, published data supported, in my clinical experience, toxicity avoidance and need for rapid response. On 29 January 2020, 
Table 1. Clinical attributes of four hypothetical metastatic triple-negative breast cancer clinical scenarios.

\begin{tabular}{|c|c|c|c|c|}
\hline Criteria & CS1 & CS2 & CS3 & CS4 \\
\hline Age & 37 & 42 & 55 & 65 \\
\hline Menopausal status & Premenopausal & Premenopausal & Postmenopausal & Postmenopausal \\
\hline Race & African-American & Caucasian & Caucasian & Hispanic \\
\hline Stage at diagnosis & T2, NO, MO & T1, N1, M1 & T1, N2, M0 & T3, N1, M1 \\
\hline Molecular diagnostics & $B R C A$ and PD-L1 negative & $\begin{array}{l}B R C A \text { negative and PD-L1 } \\
\text { positive }\end{array}$ & $\begin{array}{l}B R C A \text { negative and PD-L1 } \\
\text { positive }\end{array}$ & $B R C A$ and $\mathrm{PD}-\mathrm{L} 1$ negative \\
\hline Adjuvant therapy & $\begin{array}{l}\text { Adriamycin }+ \\
\text { cyclophosphamide followed by } \\
\text { taxol }\end{array}$ & None, de novo metastatic & TAC & None, de novo metastatic \\
\hline Time to metastatic relapse & 3 years & NA & 3 years & NA \\
\hline Presentation & $\begin{array}{l}\text { Asymptomatic; metastasis } \\
\text { observed on routine restaging }\end{array}$ & $\begin{array}{l}\text { Asymptomatic; metastasis } \\
\text { identified during staging }\end{array}$ & $\begin{array}{l}\text { Asymptomatic; metastasis } \\
\text { observed on routine restaging }\end{array}$ & $\begin{array}{l}\text { Asymptomatic; metastasis } \\
\text { identified during staging }\end{array}$ \\
\hline Sites of metastasis & Bone & Bulky liver & Lung and bone & Bulky liver \\
\hline Progression to $2 \mathrm{~L}$ & $\begin{array}{l}6 \text { months after initiation } \\
\text { therapy }\end{array}$ & $\begin{array}{l}6 \text { months after initiation } \\
\text { therapy }\end{array}$ & $\begin{array}{l}6 \text { months after initiation } \\
\text { therapy }\end{array}$ & 6 months after initiation therapy \\
\hline ECOG-PS at progression & 1 & 1 & 1 & 1 \\
\hline Post-treatment residual toxicity & None & None & None & None \\
\hline Progression to $3 \mathrm{~L}$ & 6 months & 6 months & 6 months & 6 months \\
\hline
\end{tabular}

2L: Second line; 3L: Third line; CS: Clinical scenario; ECOG-PS: Eastern Cooperative Oncology Group performance status; NA: Not appropriate; TAC: Taxotere-adriamycin-cytoxan.

the Western Institutional Review Board approved a request for a waiver of authorization for use and disclosure of protected health information for this research.

\section{Data \& analysis}

The proportion of providers selecting each treatment (specific drug/regimen) per CS is reported. Across the four CS, certain clinical features were consistent and the proportion of respondents selecting regimens per characteristic are reported. For example, in CS1 and CS3 the hypothetical patients had received adjuvant therapy, and both were without bulky liver metastases but differed in menopause status and PD-L1 expression. Thus, preferred therapy for adjuvant treatment would be reported across both CS. The chi-square test statistic was utilized to compare the proportion of providers who selected ANP versus other therapies (SA and CC combined) in each CS and/or across characteristics. A standard error and $95 \%$ CI to our point estimates were calculated to further address the imprecision due to the sample size.

\section{Results}

\section{Participants}

Seven hundred and eighty practicing oncologists who are members of the Cardinal Health Oncology Provider Extended Network, a research community, were invited to complete an eligibility screening document for participation in this research project. Eligibility requirements were: practicing in a community-based environment, spending at least $75 \%$ of their time in direct patient care and able to participate in a central institutional review board-approved study. A total of $81(10 \%)$ completed the screener, of whom 60 were selected based upon selfreported higher volumes of mTNBC patients. A total of 47 physicians completed the web-based survey within the designated time allowed and participated in this DCE. Twenty-five self-identified their primary medical specialty as hematology oncology and 22 as medical oncology whose geographic distribution was: $32 \%$ south, $28 \%$ midwest, $23 \%$ northeast and $17 \%$ west. Participants had been in practice for an average of 17.7 years with an average daily census of 22.7 patients per day. In the preceding month, they averaged 41.7 unique $\mathrm{mBC}$ patients under active management with a median of six mTNBC patients (half in later LOT; Table 2).

\section{ANP preference in $1 \mathrm{~L}$}

Overall, nearly half (47\%; 95\% CI: 40.1-54.7\%) of the participants preferred ANP in 1L, and this choice seemed irrespective of menopausal status, prior adjuvant therapy or tumor bulk at presentation of mTNBC. PD-L1 expression was the only attribute with a significant impact on ANP preference, with 69\% (95\% CI: 58.7-78.0\%) choosing ANP for those with $\geq 1 \%$ expression versus only $26 \%$ (95\% CI: $17.3-35.8 \%$ ) for those with $<1 \%$ (p 
Table 2. Participant demographics.

\begin{tabular}{|l|l|}
\hline Participant demographics & $n=47$ \\
\hline $\begin{array}{l}\text { Specialty, } \mathrm{n}(\%) \\
\text { - Hematology oncology }\end{array}$ & $25(53)$ \\
- Medical oncology & $22(47)$ \\
\hline $\begin{array}{l}\text { US region of practice, } \mathrm{n}(\%) \\
\text { - South }\end{array}$ & $15(32)$ \\
- Midwest & $13(28)$ \\
- Northeast & $11(23)$ \\
\hline - West & $8(17)$ \\
\hline Years in practice, mean (range) & $17.7(3-31)$ \\
\hline Patient volume per day, mean (range) & $22.7(5-50)$ \\
\hline Current metastatic breast cancer patient volume, mean (range) & $41.7(12-99)$ \\
\hline Number of unique patients with mTNBC treated in the previous month, median (range) & $6(1-35)$ \\
\hline mTNBC: Metastatic triple-negative breast cancer. & \\
\hline
\end{tabular}

$<0.00001)$. Conversely, choice of CC (20\%; 95\% CI: 14.9-26.8\%) or SA (32\%; 95\% CI: 25.9-39.7\%) was significantly impacted by each attribute $(\mathrm{p}<0.00001)$. SA preferred over CC in: premenopausal $(35 \% ; 95 \%$ CI: 25.7-45.7\%, vs 17\%; 95\% CI: 10.3-26.5\%; p = 0.0043737), postmenopausal (30\%; 95\% CI: 21.0-40.2\%, vs 23\%; 95\% CI: 15.5-33.5\%; p = 0.321984), adjuvant (41\%; 95\% CI: 31.6-52.1\%, vs 12\%; 6.3-20.4\%; p = 0.00026), PD-L1 <1\% (44\%; 95\% CI: 33.5-54.2\%, vs 31\%; 95\% CI: 22.0-41.3\%; p = 0.070237) and PD-L1 $\geq 1 \%$ (21\%; 95\% CI: 13.8-31.2\%, vs 10\%; 95\% CI: 4.7-17.8\%; p = 0.026342); while CC was preferred over SA in de novo metastatic presentation (29\%; 95\% CI: 20.1-39.1\%, vs 23\%; 95\% CI: $15.5-33.5 \%$; p = 0.406144) and bulky disease presentation (29\%; 95\% CI: 20.1-39.1\%, vs 23\%; 95\% CI: 15.5-33.5\%; p = 0.406144; Table 3).

\section{Reasons for $1 \mathrm{~L}$ treatment preference}

Explanations for $1 \mathrm{~L}$ treatment preference varied by treatment choice and were somewhat irrespective of the attribute. The dominant rationale chosen to explain ANP preference was 'supported by published data', mean 69\% (95\% CI: 57.7-77.7\%; range 54-74\%), while 'offering best balance of efficacy and toxicity' and 'combination therapy is appropriate when a rapid response is needed' were distant follow-up reasons for preference with, mean $12 \%(95 \%$ CI: 6.6-21.4\%) and 11\% (95\% CI: 5.8-20.1\%; Table 4). The dominant rationale for CC preference favored 'offering best balance of efficacy and toxicity' (48\%) while those preferring SA chose 'single-agent chemotherapy is recommended by guideline(s)’ (49\%).

Impact of $1 \mathrm{~L}$ preference on $2 \mathrm{~L} / 3 \mathrm{~L}$ treatment choice

Of those selecting ANP in 1L, preferences for 2L were SA 62\% (95\% CI: 50.8-71.7\%) and CC 38\% (95\% CI: 28.3-49.2\%; $\mathrm{p}=0.001644)$. CC use in $2 \mathrm{~L}$ following ANP was significantly higher than CC use following CC or SA, 38\% (95\% CI: 28.3-49.2\%) versus 18\% (95\% CI: 8.1-34.1\%) and 8\% (95\% CI: 3.0-18.3\%), respectively $(\mathrm{p}=0.000033)$. The top two preferred SA agents following ANP were capecitabine (47\%; 95\% CI: 33.9-61.1\%) and eribulin (20\%; 95\% CI: 10.9-33.4\%; $\mathrm{p}=0.002469)$; and the top two CC regimens were gemcitabine plus carboplatin (59\%; 95\% CI: 40.8-74.9\%) and doxorubicin or epirubicin plus cyclophosphamide AC/EC (24\%; 95\% CI: 11.7-42.6\%; $\mathrm{p}=0.003108)$. When SA was selected in 1L, SA was again preferred in 2L (92\%; 95\% CI: 81.2-96.9\%), dominated by eribulin (34\%; 95\% CI: 22.2-47.9\%) and capecitabine (27\%; 95\% CI: 16.2-40.5\%; $\mathrm{p}=0.411066)$. When CC was selected in $1 \mathrm{~L}$, preferences for $2 \mathrm{~L}$ were SA (82\%; 95\% CI: 65.1-91.7\%), and again, led by capecitabine (29\%; 95\% CI: 14.9-48.2\%) and eribulin (13\%; 95\% CI: 4.2-30.8\%; p = 0.118784). SA use dominated $3 \mathrm{~L}(91 \%)$ regardless of $1 \mathrm{~L}$ therapy selection with eribulin and capecitabine retaining preference, but now closely followed by gemcitabine (Table 5).

\section{Discussion}

Historically, most novel cancer drugs gain initial approval for refractory metastatic disease, and only after years on the market, expand indications to $1 \mathrm{~L}$. The advent of targeted and IO therapies with unique mechanisms of action has altered that paradigm, bringing drugs and regimens to market with 1L indication at initial US FDA approval and with which prescribing physicians have limited, if any, management experience. ANP for mTNBC is such a situation. To understand the adoption of ANP and the attributes that impact prescriber selection, we 


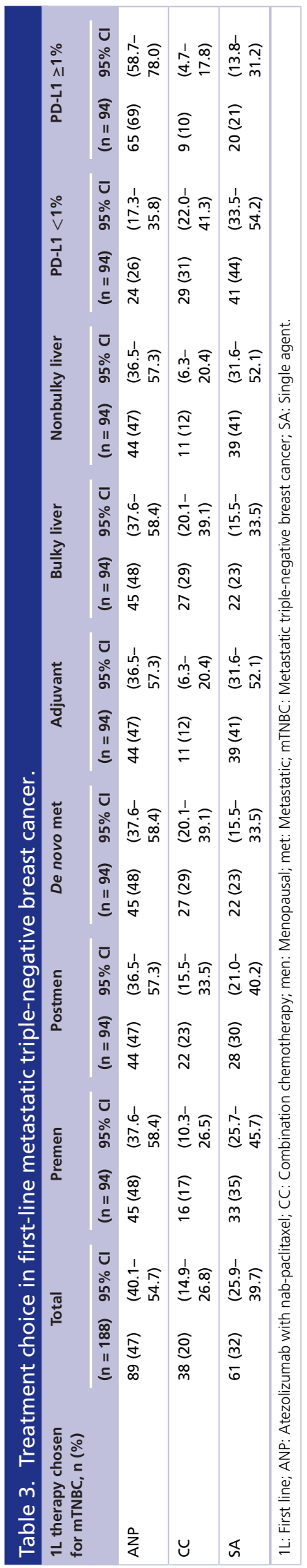


Research Article Feinberg, Hime, Wojtynek et al.

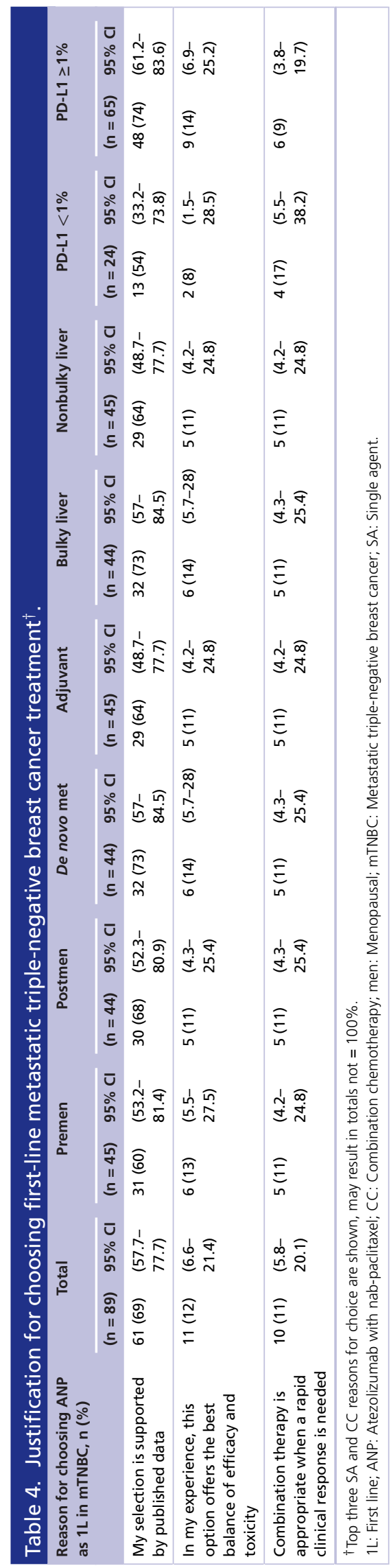


Table 5. Second- and third-line treatment choice ${ }^{\dagger}$.

\begin{tabular}{|c|c|c|c|c|c|c|c|}
\hline \multicolumn{2}{|c|}{ mTNBC sequence, $n(\%)$} & \multicolumn{6}{|c|}{ 1L } \\
\hline & & \multicolumn{2}{|c|}{ ANP } & \multicolumn{2}{|c|}{ CC } & \multicolumn{2}{|c|}{ SA } \\
\hline & & $(n=89)$ & $95 \% \mathrm{Cl}$ & $(n=38)$ & $95 \% \mathrm{Cl}$ & $(n=61)$ & $95 \% \mathrm{Cl}$ \\
\hline \multirow[t]{8}{*}{$2 \mathrm{~L}$} & SA & $55(62)$ & $(50.8-71.7)$ & $31(82)$ & $(65.1-91.7)$ & $56(92)$ & $(81.2-96.9)$ \\
\hline & - Capecitabine & $26(47)$ & $(33.9-61.1)$ & $9(29)$ & $(14.9-48.2)$ & $15(27)$ & $(16.2-40.5)$ \\
\hline & -Eribulin & $11(20)$ & $(10.9-33.4)$ & $4(13)$ & $(4.2-30.8)$ & 19 (34) & $(22.2-47.9)$ \\
\hline & - Gemcitabine & $4(7)$ & $(2.3-17.8)$ & $0(0)$ & - & $5(7)$ & $(2.6-16.3)$ \\
\hline & CC & $34(38)$ & $(28.3-49.2)$ & $7(18)$ & $(8.1-34.1)$ & $5(8)$ & (3.0-18.3) \\
\hline & $-G C$ & $20(59)$ & $(40.8-74.9)$ & $1(14)$ & $(0.8-58)$ & $4(80)$ & $(29.9-98.9)$ \\
\hline & - ANP & $2(6)$ & $(1.1-21.6)$ & $3(43)$ & $(11.8-79.8)$ & $1(20)$ & $(1.1-70.1)$ \\
\hline & - Dox/Epi+AC/EC & $8(24)$ & $(11.7-42.6)$ & $1(14)$ & $(0.8-58)$ & $0(0)$ & - \\
\hline \multirow[t]{8}{*}{$3 \mathrm{~L}$} & SA & $82(92)$ & $(83.9-96.5)$ & $32(84)$ & $(68.1-93.4)$ & $54(89)$ & $(77.2-94.9)$ \\
\hline & -Eribulin & $34(41)$ & $(30.5-52.3)$ & $11(34)$ & $(19.2-53.2)$ & $14(26)$ & (15.4-39.9) \\
\hline & - Capecitabine & $15(18)$ & $(10.8-28.4)$ & $10(31)$ & $(16.7-50.1)$ & $12(22)$ & $(12.2-35.4)$ \\
\hline & - Gemcitabine & $13(16)$ & $(9.2-26.2)$ & $4(13)$ & $(4.2-30.8)$ & $10(19)$ & $(9.9-32.4)$ \\
\hline & CC & $7(8)$ & $(3.5-16.2)$ & $6(16)$ & $(6.6-31.9)$ & $7(11)$ & $(4.9-21.8)$ \\
\hline & $-A N P$ & $4(57)$ & $(20.2-88.2)$ & $3(50)$ & $(13.9-86.1)$ & $2(29)$ & $(5.1-69.7)$ \\
\hline & $-\mathrm{GC}$ & $1(14)$ & $(0.8-58)$ & $1(17)$ & $(0.9-63.5)$ & $4(57)$ & $(20.2-88.2)$ \\
\hline & - Dox/Epi+AC/EC & $0(0)$ & - & $1(17)$ & $(0.9-63.5)$ & $1(14)$ & $(0.8-58)$ \\
\hline
\end{tabular}

chose to evaluate the relative influence of patient, tumor and clinical attributes on physicians' preferences when prescribing 1L ANP for patients with $\mathrm{mTNBC}$ using a DCE. The attributes we assessed were menopause status, prior adjuvant therapy, tumor bulk in the initial presentation of metastatic disease and PD-L1 expression. We chose menopause status as a surrogate for age grouping (e.g., $<50$ vs $>50$ years), as it has been suggested that physicians are more aggressive in their management of younger patients. We chose bulky liver metastases as a surrogate for high visceral disease burden, which has been used in clinical guidelines as an indicator to consider more aggressive therapy. We chose adjuvant chemotherapy with a taxane to evaluate prior taxane use on ANP prescribing. Other clinical variables were addressed through consistency in CS with disease-free intervals of 3 years for both adjuvant CS, all patients being asymptomatic at presentation, which is a surrogate for Eastern Cooperative Oncology Group performance status 0,1 and all disease being found on routine surveillance/staging. We also specified that each patient have an Eastern Cooperative Oncology Group performance status of 1 and no residual toxicity at each presentation (Table 1).

DCE is a powerful multidimensional tool that can be used to analyze simultaneously the importance of multiple factors on medical decision making, which would not be possible using unidimensional measures such as Likert scales [7]. Despite the relative simplicity of the scenarios proposed in our questionnaire, each attribute and modality had an effect on treatment preference. The DCE is also an appropriate method to test hypothetical scenarios that could be used to assess the preferences for subsequent treatment (SA vs $\mathrm{CC}$ ) in $2 \mathrm{~L}$ and $3 \mathrm{~L}$ regarding $1 \mathrm{~L}$ ANP use.

The primary aim of this research was to identify whether there were key characteristics and details of hypothetical vignettes of $\mathrm{mTNBC}$ scenarios that would be associated with differentiated treatment selection, specifically choice of ANP. We explored several factors associated with well-accepted conceptual models and suggested to increase their realism through CS and heighten survey participant engagement via hypothetical patient vignettes. Our findings revealed a moderate level of preference for ANP in $1 \mathrm{~L}$ mTNBC ( $47 \%$ overall) comprised of $69 \%$ preference in patients with PD-L1 expression $\geq 1 \%$ and $26 \%$ preference when expression $<1 \%$. The choice of 'observance of guideline recommendations' was the dominant explanation in both cohorts, PD-L1 <1\% (54\%) and PD-L1 $\geq 1 \%(74 \%)$, a surprising choice for the $<1 \%$ cohort as it is actually in conflict with EBM guidelines but may be explained in published trial data. A Phase Ib study in mTNBC investigated ANP and found the overall response rate (ORR) to be $38 \%$. The study did not require tumor PD-L1 positivity, and responses were seen irrespective of PD-L1 status [11]. Another study investigating pembrolizumab + eribulin in 106 patients with mTNBC and had an ORR of $26.4 \%$ (95\% CI: 18.3-35.9\%) with response rates similar regardless of PD-L1 status [12]. Finally, 
a small study $(\mathrm{n}=12)$ evaluating pembrolizumab with capecitabine in mTNBC irrespective of PD-L1 expression found the ORR to be $42 \%$ [13].

Our results revealed a limited influence of the other clinical attributes studied on 1L ANP treatment preference, as prior adjuvant treatment, tumor bulk at initial metastatic presentation and menopause, none of these had an impact on ANP choice. This contrasts with the influence these very same attributes had on choice of SA or CC, affirming the historical importance of these attributes.

The impact of ANP on subsequent LOT was profound in one remarkable way, the use of CC in $2 \mathrm{~L}(38 \%)$, whereas 2L CC use following $1 \mathrm{~L}$ CC or 1L SA was 18 and 8\%, respectively. The predominance of SA choice in $2 \mathrm{~L}$ and $3 \mathrm{~L}$ is consistent with published guidelines. The extent of choice of CC both as an alternative to ANP in 1L (18\%), for those with PD-L1 expression <1\% (31\%), bulky liver metastases $(29 \%)$ and in subsequent LOT (2L: $8-38 \%$; 3L: $8-16 \%)$ is not guideline supported and warrants further evaluation with real-world data. The few preferred SA drugs and CC regimens were notable given the number of options that are available, guideline listed and with similar levels of evidence. SA drugs capecitabine and eribulin and CC doublets carboplatin plus gemcitabine and anthracycline plus cyclophosphamide were the dominant choices in aggregate and for each of the LOT studied and irrespective of clinical attributes.

Several limitations of the study should be noted. In particular, this manuscript is descriptive in nature and is being utilized for further hypotheses to be generated. The results are describing the observations among the participants $(\mathrm{n}=47)$ only, and no formal hypothesis is being tested. We have added a standard error and $95 \%$ CI to our point estimates to further address the imprecision due to the sample size. Also, the limited number of attributes and scenarios tested do not reflect the full complexity of medical decision making in $\mathrm{mBC}$. Indeed, the factors impacting choice as described earlier are myriad. Analyses were limited to selected patients for whom recent guideline changes may have resulted in confusion regarding the evidence to guide decision making. This issue underlines a classical limitation of the DCE for which a limited number of attributes and modalities are required in terms of feasibility. Long and complex questionnaires lead to cognitive limitations and missing data. In this respect, the results of the chosen design allowed us to complete the DCE and gather insight into physician prescribing preference but not identify other factors such as patient's preference, complexity of therapeutic protocols and financial implications, among the many prior noted variables that also influence treatment choice.

This research suggests that ANP in $1 \mathrm{~L}$ PD-L1 $\geq 1 \% \mathrm{mTNBC}$ has been rapidly adopted as a standard of care. The $26 \%$ use in patients with PD-L1 expression $<1 \%$ warrants provider-directed education. The use of CC as an alternative to ANP in $1 \mathrm{~L}$, particularly in bulky liver metastases and in $2 \mathrm{~L}$, warrants greater guideline specificity in the IO era.

\section{Future perspective}

EBM in the selection of $\mathrm{mBC}$ treatment is somewhat aspirational as initial and subsequent treatment selections are influenced by myriad nonclinical payer, provider, patient and societal factors, for example, benefit design, heuristics, health literacy and social determinants of health. DCE are among the increasingly valuable research techniques that will be critical given the limitations of traditional clinical trial research. Real-world data sources, patient-reported outcomes analyses, comparative effectiveness research and DCE are just a few of the expansive arsenal of tools that will become routine in the assessment of treatment preference and value that will complement and supplement traditional clinical trial research findings. This is possibly more needed in oncology than other specialty areas of medicine given the increasing niche patient populations defined by molecular subtypes, the explosive development of new therapies, the $<5 \%$ accrual of adult US cancer patients to clinical trials and their non-representative nature as those that are accrued are younger, healthier and less diverse than those treated off trial.

\section{Conclusion}

DCE represents a valuable research tool to better understand real-world physician treatment preferences and provides unique insights into patient care not available in clinical trials. The influences on treatment selection are far more complex than the clinical trial end points of efficacy and toxicity. Our research into physician treatment preference for $\mathrm{mBC}$ revealed a lack of uniformity among oncologists and was not always consistent with published guidelines. We believe this kind of research will be increasingly critical to understand the complexities of prescribing behavior as cancer care transforms to a value-based care structure. 
Summary points

- The practice of evidence-based medicine is somewhat aspirational as there are myriad influences on physician and patient treatment choice.

- The management of metastatic breast cancer $(\mathrm{mBC})$ is particularly complex as the range of evidence-based medicine choices is both dynamic and wide.

- That complexity is further challenged by recent guideline recommendation changes to first-line (1L) treatment of triple-negative $\mathrm{mBC}$ in which atezolizumab with nab-paclitaxel (ANP) is preferred for patients with PD-L1 expression $\geq 1 \%$.

- We conducted a discrete choice experiment in which common triple-negative $\mathrm{mBC}$ patient clinical scenarios were presented as case studies with participant physicians queried regarding choice of ANP in 1L.

- The impact on treatment preference was assessed for four attributes in $1 \mathrm{~L} \mathrm{mBC}$ : prior adjuvant therapy, bulky disease presentation, PD-L1 expression $\geq 1 \%$ and menopause status.

- We also evaluated how $1 \mathrm{~L}$ choice impacted second-line and third-line choices.

- We queried the rationale for all choices.

- Choice of ANP overall was $47 \%$ in those with PD-L1 expression $\geq 1 \%(69 \%)$ and $<1 \%(26 \%)$. Choice of combination chemotherapy in $1 \mathrm{~L}$ and beyond warrants further exploration as such choices deviate from guideline recommendations.

Financial \& competing interests disclosure

This study was funded by Athenex, Inc., Buffalo, NY, USA. The authors have no other relevant affiliations or financial involvement with any organization or entity with a financial interest in or financial conflict with the subject matter or materials discussed in the manuscript apart from those disclosed.

No writing assistance was utilized in the production of this manuscript.

Ethical conduct of research

On 29 January 2020, Western Institutional Review Board (WIRB) approved a request for a waiver of authorization for use and disclosure of protected health information (PHI) for this research.

\section{Data sharing statement}

Datasets generated during and/or analyzed during the current study will not be deposited.

\section{Open access}

This work is licensed under the Attribution-NonCommercial-NoDerivatives 4.0 Unported License. To view a copy of this license, visit http://creativecommons.org/licenses/by-nc-nd/4.0/

\section{References}

Papers of special note have been highlighted as: $\bullet$ of interest; $\bullet \bullet$ of considerable interest

1. Mestres JA, iMolins AB, Martínez LC et al. Defining the optimal sequence for the systemic treatment of metastatic breast cancer. Clin. Transl. Oncol. 19, 149-161 (2017).

-• After discussion by specialized breast cancer oncologists, a general consensus of treatment patterns based on patient characteristics and previous lines of therapy was reached.

2. Benjamin L, Cotté FE, Philippe $\mathrm{C}$ et al. Physicians' preferences for prescribing oral and intravenous anticancer drugs: a discrete choice experiment. Eur. J. Cancer 48(6), 912-920 (2012).

-. A discrete choice experiment (DCE) was conducted to elicit treatment preferences. Results suggest that economic considerations and therapeutic efficacy play a significant role in choice of treatment.

3. MacEwan JP, Doctor J, Mulligan K et al. The value of progression-free survival in metastatic breast cancer: results from a survey of patients and providers. MDM Policy Pract. 4(1), 1-14 (2019).

- This study evaluated the preference for progression-free survival as a clinical trial endpoint in metastatic breast cancer. Results suggest a strong preference for progression-free survival as well as a range of endpoints in clinical trials.

4. Guerra RL, Castaneda L, de Albuquerque RCR et al. Patient preferences for breast cancer treatment interventions: a systematic review of discrete choice experiments. Patient 12(6), 559-569 (2019).

- This review summarizes patient preferences for breast cancer treatments via DCE. Results suggest that patients value greater benefit and are willing to risk side effects for gains in survival.

5. Phillips CM, Deal K, Powis M et al. Evaluating patients' perception of the risk of acute cancer visits during systemic therapy for cancer. JCO Oncol. Pract. 16(7), e622-e629 (2020). 
6. Gonzalez JM, Ogale S, Morlock R et al. Patient and physician preferences for anticancer drugs for the treatment of metastatic colorectal cancer: a discrete-choice experiment. Cancer Manag. Res. 9, 149-158 (2017).

- This study evaluates preferences for anticancer drugs in metastatic colorectal cancer using a DCE. There is a difference in preference between patients and physicians, suggesting there is potential for improvement in patients' well-being.

7. Persky S, Kaphingst KA, Condit CM et al. Assessing hypothetical scenario methodology in genetic susceptibility testing analog studies: a quantitative review. Genet. Med. 9, 727-738 (2007).

-• This review explores multiple theoretically based factors on genetic test uptake accuracy using hypothetical scenario methodology.

8. Liu FX, Witt EA, Ebbinghaus S et al. Patient and oncologist preferences for attributes of treatments in advanced melanoma: a discrete choice experiment. Patient Prefer. Adherence 11, 1389-1399 (2017).

9. Lin PL, Hao Y, Xie J et al. Physician experiences and preferences in the treatment of HR+/HER2- metastatic breast cancer in the United States: a physician survey. Cancer Med. 5(2), 209-220 (2016).

10. Rocque GB, Rasool A, Williams BR et al. What is important when making treatment decisions in metastatic breast cancer? A qualitative analysis of decision-making in patients and oncologists. Oncologist 24, 1313-1321 (2019).

- This analysis describes patient and provider factors that influence the decision-making process in treating metastatic breast cancer.

11. Adams S, Diamond JR, Hamilton EP. Phase Ib trial of atezolizumab in combination with nab-paclitaxel in patients with metastatic triple-negative breast cancer (mTNBC). J. Clin. Oncol. 34(Suppl. 15), Abstract 1009 (2016).

12. Tolaney SM, Kalinsky K, Kaklamani V. Phase Ib/II study to evaluate eribulin mesylate in combination with pembrolizumab in patients with metastatic triple-negative breast cancer. Cancer Res. 78(4), Abstract PD6-13 (2018).

13. Page DB, Kim IK, Sanchez K. Safety and efficacy of pembrolizumab (pembro) plus capecitabine (cape) in metastatic triple negative breast cancer (mTNBC). J. Clin. Oncol. 36(Suppl. 15), Abstract 1033 (2018). 